\title{
About the Frequencies of Some Patterns in Digital Planes Application to Area Estimators
}

\author{
Alain Daurat, Mohamed Tajine, and Mahdi Zouaoui \\ LSIIT CNRS UMR 7005, Université Louis Pasteur (Strasbourg 1), \\ Pôle API, Boulevard Sébastien Brant, 67400 Illkirch-Graffenstaden, France \\ \{daurat, tajine, zouaoui\}@dpt-info.u-strasbg.fr
}

\begin{abstract}
In this paper we prove that the function giving the frequency of a class of patterns of digital planes with respect to the slopes of the plane is continuous and piecewise affine, moreover the regions of affinity are precised. It allows to prove some combinatorial properties of a class of patterns called $(m, n)$-cubes. This study has also some consequences on local estimators of area: we prove that the local estimators restricted to regions of plane never converge to the exact area when the resolution tends to zero for almost all slope of plane. Actually all the results of this paper can be generalized for the regions of hyperplanes for any dimension $d \geq 3$.

The proofs of some results used in this article are contained in the extended version of this paper [1].
\end{abstract}

Keywords: Digital Plane, Pattern, $(m, n)$-cube, Area Estimator, Local Estimator, Multigrid Convergence.

\section{Introduction}

Digital Planes are very classical objects of Discrete Geometry. Their combinatorics have been studied in a lot of papers (for example $2,2,3,4,5,6,7,8,9,10,11$, 12, 13.), for a recent review on the subject, see 14. In this paper we are interested in a class of patterns called $(m, n)$-cubes which are intuitively the pieces of digital planes of size $m \times n$. These objects have been studied for quite a long time, for example it is well-known that the number of $(m, n)$-cubes appearing in a digital plane is always less than $m n$ ( [5, 6, 7, 9, 11]). These $(m, n)$-cubes can be used in different domains of image analysis for example for normal vector estimation, area estimation ( [15, 16, see also second section of this paper), form reconstruction. The originality of this paper is the study of not only the presence of a $(m, n)$-cube in a digital plane, but also of the frequency of this $(m, n)$-cube in all the digital planes.

The main result of the first part of this paper enunciates that the function giving the frequency of $(m, n)$-cubes of digital planes with respect to the slopes of the plane is continuous and piecewise affine. Moreover we will see that the study of the frequency allows to prove some combinatorial properties on the $(m, n)$-cubes.

D. Coeurjolly et al. (Eds.): DGCI 2008, LNCS 4992, pp. 45 56, 2008.

(C) Springer-Verlag Berlin Heidelberg 2008 
In a second part of the paper, we use our study about frequencies to prove some results about local estimators of area. A local estimator of area simply consists to decompose a surface into little pieces (in fact similar to $(m, n)$-cubes) and to sum some weights which correspond to the pieces. The study of the frequency of the $(m, n)$-cubes allows to prove that even for planar regions these estimators are not correct in the sense that, if the discrete regions are obtained from a continuous plane, then the estimated area does not converge to the exact area for almost all slopes of plane when the resolution of the discretization tends to zero. It is in fact a generalization to $3 \mathrm{D}$ of [17. Actually we can prove with the same technics that all the results of this paper are true for the regions of hyperplanes for any dimension $d \geq 3$.

The proofs of some results used in this article are contained in the extended version of this paper [1.

\section{Preliminaries}

Let $a, b \in \mathbb{N}$ and $a \leq b$. The discrete interval $\{a, a+1, \ldots, b-1, b\}$ is denoted $\llbracket a, b \rrbracket$. For $x \in \mathbb{R},\lfloor x\rfloor$ (resp. $\langle x\rangle$ ) denotes the integral part (resp. the fractional part) of $x$. So, $x=\lfloor x\rfloor+\langle x\rangle$ with $\lfloor x\rfloor \in \mathbb{Z},\lfloor x\rfloor \leq x<\lfloor x\rfloor+1$ and $0 \leq\langle x\rangle<1$. For any set $E, \operatorname{card}(E)$ denotes the cardinality of $E$.

We refer in all the following to a subset of $\mathbb{R}^{3}$ of the form $R=\{(x, y, \alpha x+$ $\beta y+\gamma) \mid a \leq x \leq b$ and $c \leq y \leq d\}$ such that $\alpha, \beta \in[0,1]$ and $a, b, c, d \in \mathbb{R}$ as a rectangular planar region. It corresponds to a subset of plane whose projection on the $X Y$-plane is a rectangle with sides parallel to $X, Y$-axes.

In this paper all the topological notions are considered relatively to the Euclidean usual topology. If $E$ is subset of a topological space, $\bar{E}$ denotes its topological closure (the smallest closed set containing $E$ ). The measure notions are considered relatively to the Lebesgue measure on the Euclidean space, for example 'negligible set' (set with zero measure) and 'almost everywhere' are considered relatively to the Lebesgue measure on the Euclidean space.

\section{Frequencies of the $(m, n)$-cubes}

In this paper we consider naive digital planes $P_{\alpha, \beta, \gamma}=\{(x, y,\lfloor\alpha x+\beta y+$ $\left.\gamma\rfloor) \mid(x, y) \in \mathbb{Z}^{2}\right\}$ with $\alpha, \beta \in[0,1]$ and $\gamma \in \mathbb{R}$. So a naive plane is functional in its $x, y$ coordinates: $z=p_{\alpha, \beta, \gamma}(x, y)=\lfloor\alpha x+\beta y+\gamma\rfloor$ for all $(x, y, z) \in P_{\alpha, \beta, \gamma}$. Moreover we fix two positive integers $m$ and $n$ and we define $\mathcal{F}_{m, n}=\llbracket 0, m-$ $1 \rrbracket \times \llbracket 0, n-1 \rrbracket$.

Definition 1. A $(m, n)$-pattern is a function $w: \mathcal{F}_{m, n} \rightarrow \mathbb{Z}$. We note $\mathcal{M}_{m, n}$ the set of all $(m, n)$-patterns and the size of $a(m, n)$-pattern is $m \times n$.

We can also see a $(m, n)$-pattern as a set of voxels which projection in the $X Y$ plane is $\mathcal{F}_{m, n}$ and which has at most one point in each line parallel to the third coordinate direction. 
In all the following, a pattern of size less than $m \times n$ corresponds to a $\left(m^{\prime}, n^{\prime}\right)$ pattern where $m^{\prime} \leq m, n^{\prime} \leq n$ and $(m, n) \neq\left(m^{\prime}, n^{\prime}\right)$.

A $(m, n)$-cube is a $(m, n)$-pattern which can be extracted from a naive digital plane, more precisely:

Definition 2. The $(m, n)$-cube at position $(i, j)$ of the digital plane $P_{\alpha, \beta, \gamma}$ is the $(m, n)$-pattern $w$ defined by $w\left(i^{\prime}, j^{\prime}\right)=p_{\alpha, \beta, \gamma}\left(i+i^{\prime}, j+j^{\prime}\right)-p_{\alpha, \beta, \gamma}(i, j)$ for any $\left(i^{\prime}, j^{\prime}\right) \in \mathcal{F}_{m, n}$. It is denoted $w_{i, j}(\alpha, \beta, \gamma)$.

So a $(m, n)$-cube is simply a piece of a digital plane which projection in the $X Y$-plane is a translation of $\mathcal{F}_{m, n}$. Fig 1 corresponds to a $(3,3)$-cube in a digital plane.

Note that for all $i, j \in \mathbb{Z}$ and $\alpha, \beta, \gamma \in \mathbb{R}, w_{i, j}(\alpha, \beta, \gamma)=w_{0,0}(\alpha, \beta, \alpha i+\beta j+\gamma)$.

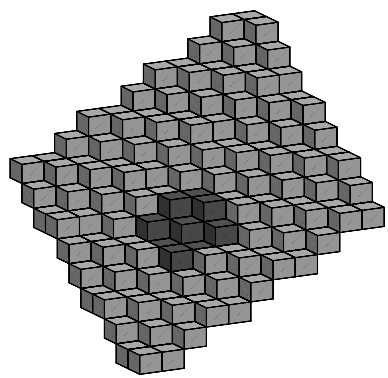

Fig. 1. A (3,3)-cube in a digital plane

Let $C_{i, j}^{\alpha, \beta}=1-\langle\alpha i+\beta j\rangle$ for $(i, j) \in \mathcal{F}_{m, n}$, and $\sigma^{\alpha, \beta}$ be a bijection from $\llbracket 1, m n \rrbracket$ to $\mathcal{F}_{m, n}$ such that the sequence $\left(B_{i}^{\alpha, \beta}\right)_{0 \leq i \leq m n}$ defined by $B_{i}^{\alpha, \beta}=C_{\sigma^{\alpha, \beta}(i)}^{\alpha, \beta}$ for $1 \leq i \leq m n$ and $B_{0}^{\alpha, \beta}=0$, is increasing.

We recall some known results (see for example [6]).

Proposition 1. For all $\alpha, \beta, \gamma \in \mathbb{R}$ we have:

1. The $(k, l)$-th point of the $(m, n)$-cube at position $(i, j)$ of the digital plane $P_{\alpha, \beta, \gamma}$ can be computed by the formula:

$$
w_{i, j}(\alpha, \beta, \gamma)(k, l)= \begin{cases}\lfloor\alpha k+\beta l\rfloor & \text { if }\langle\alpha i+\beta j+\gamma\rangle<C_{k, l}^{\alpha, \beta} \\ \lfloor\alpha k+\beta l\rfloor+1 & \text { otherwise }\end{cases}
$$

2. The $(m, n)$-cube $w_{i, j}(\alpha, \beta, \gamma)$ only depends on the interval $\left[B_{h}^{\alpha, \beta}, B_{h+1}^{\alpha, \beta}[\right.$ containing $\langle\alpha i+\beta j+\gamma\rangle$.

3. For all $h \in \llbracket 1, m n-1 \rrbracket$, if $\left[B_{h}^{\alpha, \beta}, B_{h+1}^{\alpha, \beta}\left[\right.\right.$ is not empty $\left(B_{h}^{\alpha, \beta}<B_{h+1}^{\alpha, \beta}\right)$, then there exist $i, j$ such that $\langle\alpha i+\beta j+\gamma\rangle \in\left[B_{h}^{\alpha, \beta}, B_{h+1}^{\alpha, \beta}[\right.$ and thus the number of $(m, n)$-cubes in the digital plane $P_{\alpha, \beta, \gamma}$ is equal to $\operatorname{card}\left(\left\{C_{k, l}^{\alpha, \beta} \mid(k, l) \in\right.\right.$ $\left.\left.\mathcal{F}_{m, n}\right\}\right)$. We have, in particular, $\operatorname{card}\left(\left\{C_{k, l}^{\alpha, \beta} \mid(k, l) \in \mathcal{F}_{m, n}\right\}\right) \leq m n$. 
So, we have $w_{0,0}(\alpha, \beta, \gamma)=w_{0,0}(\alpha, \beta,\langle\gamma\rangle)$ and thus $w_{i, j}(\alpha, \beta, \gamma)=w_{0,0}(\alpha, \beta,\langle\alpha i+$ $\beta j+\gamma\rangle)$ for all $\alpha, \beta, \gamma \in \mathbb{R}$ and $(i, j) \in \mathbb{Z}^{2}$.

By Proposition 1, the set of $(m, n)$-cubes of the digital plane $P_{\alpha, \beta, \gamma}$ depends only on $\alpha, \beta$ and it is denoted $\mathcal{C}_{m, n, \alpha, \beta}$. In all the following, $\mathcal{U}_{m, n}$ denotes the set of all the $(m, n)$-cubes. So, $\mathcal{U}_{m, n}=\bigcup_{(\alpha, \beta) \in[0,1]^{2}} \mathcal{C}_{m, n, \alpha, \beta}$.

Definition 3 ( [18]). Let $w$ be a $(m, n)$-cube, then the pre-image $\mathrm{PI}(w)$ of $w$ is the set of the triple $(\alpha, \beta, \gamma) \in[0,1]^{3}$ such that $w$ is the $(m, n)$-cube at position $(0,0)$ of the digital plane $P_{\alpha, \beta, \gamma}$.

Remark. It is easy to see that $\mathrm{PI}(w)$ is a convex polyhedron defined by the inequalities $w(k, l) \leq k \alpha+l \beta+\gamma<w(k, l)+1$ for $(k, l) \in \mathcal{F}_{m, n}$. Moreover the set of the $\gamma^{\prime} \in[0,1]$ such that $\left(\alpha, \beta, \gamma^{\prime}\right) \in \operatorname{PI}\left(w_{i, j}(\alpha, \beta, \gamma)\right)$ is exactly the interval $\left[B_{h}^{\alpha, \beta}, B_{h+1}^{\alpha, \beta}[\right.$ containing $\langle\alpha i+\beta j+\gamma\rangle$.

The last remark leads to the following definition:

Definition 4. The $\gamma$-frequency of a $(m, n)$-cube $w$ for the slopes $(\alpha, \beta)$ (denoted freq $\left._{\alpha, \beta}(w)\right)$ is the length of the interval $I^{\alpha, \beta}(w)=\{\gamma \in[0,1] \mid(\alpha, \beta, \gamma) \in \operatorname{PI}(w)\}$. (so the function $T P: \operatorname{PI}(w) \rightarrow \mathbb{R}$ such that $T P(\alpha, \beta)=\operatorname{freq}_{\alpha, \beta}(w)$ is the tomographic projection of $\mathrm{PI}(w)$ w.r.t. the third coordinate direction).

Definition 5. The overlapping frequency of a $(m, n)$-cube in the digital plane $P_{\alpha, \beta, \gamma}$ is

$$
\lim _{N \rightarrow+\infty} \frac{\operatorname{card}\left(\left\{(i, j) \in \llbracket-N, N \rrbracket^{2} \mid w_{i, j}(\alpha, \beta, \gamma)=w\right\}\right)}{(2 N+1)^{2}}
$$

if the limit exists. It is denoted overfreq $_{\alpha, \beta, \gamma}(w)$.

So, overfreq ${ }_{\alpha, \beta, \gamma}(w)=\lim _{N \rightarrow+\infty} \frac{\operatorname{card}\left(\left\{(i, j) \in \llbracket-N, N \rrbracket^{2} \mid\langle\alpha i+\beta j+\gamma\rangle \in I^{\alpha, \beta}(w)\right\}\right)}{(2 N+1)^{2}}$

We have the following properties:

Proposition 2 ( [1]). For any $\alpha, \beta \in[0,1]$ and $\gamma \in \mathbb{R}$ we have:

1. $w \in \mathcal{C}_{m, n, \alpha, \beta}$ if and only if $\operatorname{freq}_{\alpha, \beta}(w)>0$.

2. overfreq $_{\alpha, \beta, \gamma}(w)=\operatorname{freq}_{\alpha, \beta}(w)$

(A related result is stated in [19]).

Definition 6. A function $f: \mathbb{R}^{2} \rightarrow \mathbb{R}$ is called a piecewise affine function if there exists a finite collection $\left(C_{i}\right)_{i \in I}$ of open convex subsets of $\mathbb{R}^{2}$ and affine functions $f_{i}: \mathbb{R}^{2} \rightarrow \mathbb{R}$ for $i \in I$, such that :

$-C_{i} \cap \underline{C_{i^{\prime}}}=\emptyset$ for $i, i^{\prime} \in I$ and $i \neq i^{\prime}$,

$-\bigcup_{i \in I} \overline{C_{i}}=\mathbb{R}^{2}$ and

- the restriction of $f$ to $C_{i}$ is $f_{i}$ for all $i \in I$ (for all $i \in I, f(x)=f_{i}(x)$ for all $\left.x \in C_{i}\right)$.

Property 1 ( [1]). Let $f, g: \mathbb{R}^{2} \rightarrow \mathbb{R}$ be two piecewise affine functions. Then $-f, f+g, f-g, \max (f, g)$ and $\min (f, g)$ are also piecewise affine functions. 
Theorem 1. For any $(m, n)$-cube $w$, the function $(\alpha, \beta) \mapsto \operatorname{freq}_{\alpha, \beta}(w)$ is a continuous function which is piecewise affine.

Proof. $P I(w)=\left\{(\alpha, \beta, \gamma) \in \mathbb{R}^{3} \mid w(k, l) \leq \alpha k+\beta l+\gamma<w(k, l)+1\right.$ for $\quad$ all $(k, l)$ $\left.\in \mathcal{F}_{m, n}\right\}$.

Then $I^{\alpha, \beta}(w)=\left[\max _{(k, l) \in \mathcal{F}_{m, n}}(w(k, l)-\alpha k-\beta l), \min _{(k, l) \in \mathcal{F}_{m, n}}(w(k, l)+1-\right.$ $\alpha k-\beta l)[$.

So, $\operatorname{freq}_{\alpha, \beta}(w)=\max \left(0, \min _{(k, l) \in \mathcal{F}_{m, n}}(w(k, l)+1-\alpha k-\beta l)-\max _{(k, l) \in \mathcal{F}_{m, n}}\right.$ $(w(k, l)-\alpha k-\beta l))$. Affine functions, max and min are continuous functions. Then $(\alpha, \beta) \mapsto \operatorname{freq}_{\alpha, \beta}(w)$ is a continuous function which is piecewise affine because it is composition of continuous functions and by Property 1 it is piecewise affine function.

Proposition 3. Let $\left(\alpha_{1}, \beta_{1}\right),\left(\alpha_{2}, \beta_{2}\right),\left(\alpha_{3}, \beta_{3}\right)$ be points of $[0,1]^{2}$ and $T$ be the convex hull of these three points. Let $\left(\alpha_{0}, \beta_{0}\right) \in T$ and consider $\lambda_{1}, \lambda_{2}, \lambda_{3} \geq 0$ such that $\left(\alpha_{0}, \beta_{0}\right)=\sum_{i=1}^{3} \lambda_{i}\left(\alpha_{i}, \beta_{i}\right)$ and $\sum_{i=1}^{3} \lambda_{i}=1\left(\lambda_{1}, \lambda_{2}, \lambda_{3}\right.$ are barycentric coordinates of $\left(\alpha_{0}, \beta_{0}\right)$ relatively to $\left.\left(\alpha_{1}, \beta_{1}\right), \ldots\left(\alpha_{3}, \beta_{3}\right)\right)$. Suppose moreover that the function $(\alpha, \beta) \mapsto$ freq $_{\alpha, \beta}(w)$ is affine on $T$ for any $(m, n)$-cube $w$, then

$$
\mathcal{C}_{m, n, \alpha_{0}, \beta_{0}}=\bigcup_{1 \leq i \leq 3} \mathcal{C}_{m, n, \alpha_{i}, \beta_{i}}
$$

Proof. By affinity of $(\alpha, \beta) \mapsto \operatorname{freq}_{\alpha, \beta}(w)$ on $T$ we have:

$$
\text { freq }_{\alpha_{0}, \beta_{0}}(w)=\sum_{i=1}^{3} \lambda_{i} \operatorname{freq}_{\alpha_{i}, \beta_{i}}(w)
$$

If $w \notin \mathcal{C}_{m, n, \alpha_{0}, \beta_{0}}$ then by Proposition 2, freq $\operatorname{son}_{\alpha_{0}, \beta_{0}}(w)=0$ and so for any $i$, freq $_{\alpha_{i}, \beta_{i}}(w)=0$ or $\lambda_{i}=0$ because $\lambda_{1}, \lambda_{2}, \lambda_{3} \geq 0$ which implies that for any $i$, if $\lambda_{i} \neq 0$ then $w \notin \mathcal{C}_{m, n, \alpha_{i}, \beta_{i}}$. Conversely as $\lambda_{1}, \lambda_{2}, \lambda_{3} \geq 0$ and $\sum_{i=1}^{3} \lambda_{i}=1$, if $w \in \mathcal{C}_{m, n, \alpha_{0}, \beta_{0}}$, then by Proposition 2. freq fro $_{\alpha_{0}, \beta_{0}}(w)>0$ and thus, there must exist a $i \in\{1,2,3\}$ such that $\lambda_{i} \neq 0$ and freq $_{\alpha_{i}, \beta_{i}}(w)>0$

We will now precise the domains where the function $(\alpha, \beta) \mapsto \operatorname{freq}_{\alpha, \beta}(w)$ is affine: Let $D_{u, v, w}$ be the line $\left\{(\alpha, \beta) \in \mathbb{R}^{2} \mid \alpha u+\beta v+w=0\right\}$ and

$$
E_{m, n}=\bigcup_{(u, v, w) \in \llbracket-m+1, m-1 \rrbracket \times \llbracket-n+1, n-1 \rrbracket \times \mathbb{Z}} D_{u, v, w} \cap[0,1]^{2} .
$$

$E_{m, n}$ involves only straight lines $D_{u, v, w}$ such that $D_{u, v, w} \cap[0,1]^{2} \neq \emptyset$ and so we must only consider the straight lines $D_{u, v, w}$ such that $|w| \leq|u|+|v|$ and thus $E_{m, n}$ involves only a finite number of straight lines.

$E_{m, n}$ is called Hyper Farey fan in [2] and Farey's diagram in [9].

Fig 2 corresponds to Farey's diagram for $m=4$ and $n=3$.

Theorem 2 ( [1]). The function $(\alpha, \beta) \mapsto \operatorname{freq}_{\alpha, \beta}(w)$ is affine on the closure of any connected component of $[0,1]^{2} \backslash E_{m, n}$ for all $w \in \mathcal{U}_{m, n}$. Moreover for any $(\alpha, \beta),\left(\alpha^{\prime}, \beta^{\prime}\right) \in[0,1]^{2} \backslash E_{m, n}: \mathcal{C}_{m, n, \alpha, \beta}=\mathcal{C}_{m, n, \alpha^{\prime}, \beta^{\prime}}$ if and only if $(\alpha, \beta)$ and $\left(\alpha^{\prime}, \beta^{\prime}\right)$ are in the same connected component of $[0,1]^{2} \backslash E_{m, n}$. 


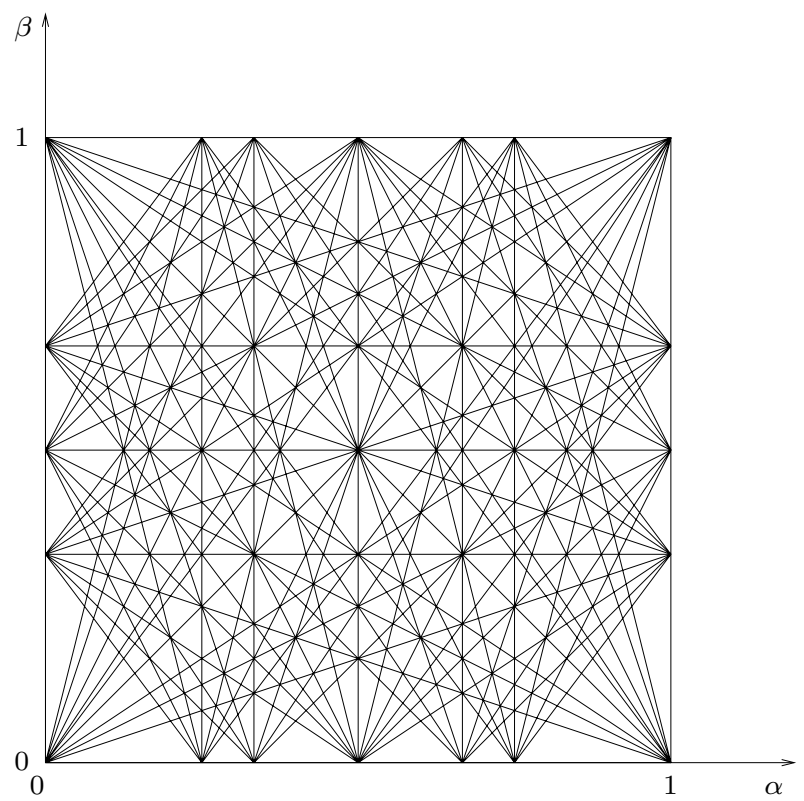

Fig. 2. Farey's diagram for $m=4$ and $n=3$

Corollary 1. Let $\mathcal{O}$ be a connected component of $[0,1]^{2} \backslash E_{m, n}$. Then $\mathcal{O}$ is a convex polygon and if $p_{1}, p_{2}, p_{3}$ are distinct vertexes of the polygon $\mathcal{O}$ then

1. for any point $p \in \mathcal{O}, \mathcal{C}_{m, n, p}=\mathcal{C}_{m, n, p_{1}} \cup \mathcal{C}_{m, n, p_{2}} \cup \mathcal{C}_{m, n, p_{3}}$ and

2. for any point $p$ in the interior of the segment of vertexes $p_{1}, p_{2}, \mathcal{C}_{m, n, p}=$ $\mathcal{C}_{m, n, p_{1}} \cup \mathcal{C}_{m, n, p_{2}}$.

Proof. The function $(\alpha, \beta) \mapsto \mathcal{C}_{m, n, \alpha, \beta}$ is constant on $\mathcal{O}$. By Theorem 2 , for all $w \in \mathcal{U}_{m, n}$, the function $(\alpha, \beta) \mapsto \operatorname{freq}_{\alpha, \beta}(w)$ is affine on $\overline{\mathcal{O}}$ and we conclude by using Proposition 3

Corollary 2. The number of $(m, n)$-cubes is in $O\left((m+n)^{2} m^{3} n^{3}\right)$.

Proof. Any line of equation $u x+v y+w=0$ with $|w|>|u|+|v|$ does not intersect the square $[0,1]^{2}$, so $E_{m, n}$ is composed of at most $(2 m+1)(2 n+1)(2(m+n+$ $1)+1)=f(m, n)$ lines. Thanks to Theorem 2 and Corollary 3 all the $(m, n)$ cubes appear in the vertices of the connected components of $[0,1]^{2} \cap E_{m, n}$. Each vertex is the intersection of two lines of $E_{m, n}$ so there are at most $f(m, n)^{2}$ such vertices. Each vertex corresponds to at most $m n(m, n)$-cubes, so in total there are at most $((2 m+1)(2 n+1)(2(m+n+1)+1))^{2} m n(m, n)$-cubes, which proves the claim.

Corollary 2 gives an upper bound for the number of $(m, n)$-cubes. In the following, we will give a lower bound for this number. 
Definition 7. Let $m \in \mathbb{N} \backslash\{0\}$.

1. Let $\alpha, \gamma \in[0,1]$. The set $S(m, \alpha, \gamma)=\{(x,\lfloor\alpha x+\gamma\rfloor) \mid x \in \llbracket 0, m-1 \rrbracket\}$ is called a digital segment of size $m$.

2. $\mathcal{S}_{m}=\{S(m, \alpha, \gamma) \mid \alpha, \gamma \in[0,1]\}$ is the set of all digital segments of size $m$.

\section{Property $2([20,21])$}

1. $\operatorname{card}\left(\mathcal{S}_{m}\right)=1+\sum_{i=1}^{m}(m-i+1) \varphi(i)$ where $\varphi$ is the Euler's totient function $(\varphi(i)=\operatorname{card}(\{j \mid 1 \leq j<i$ and $i$ and $j$ are co-prime $\}))$.

2. $\operatorname{card}\left(\mathcal{S}_{m}\right)=\frac{m^{3}}{\pi^{2}}+O\left(m^{2} \log (m)\right)$.

Property 3. Let $m, n \in \mathbb{N}^{*}$ and $\alpha, \beta, \gamma_{1}, \gamma_{2} \in[0,1]$ and consider the two digital segments $S\left(m, \alpha, \gamma_{1}\right), S\left(n, \beta, \gamma_{2}\right)$. Then, there exists $w \in \mathcal{U}_{m, n}$ such that $S\left(m, \alpha, \gamma_{1}\right)=\{(i, w(i, 0)) \mid i \in \llbracket 0, m-1 \rrbracket\}$ and $S\left(m, \beta, \gamma_{2}\right)=\{(j, w(0, j)) \mid j \in$ $\llbracket 0, m-1 \rrbracket\}$.

Proof. By [21, 6], there exist $i, j \in \mathbb{Z}$ such that $S\left(m, \alpha, \gamma_{1}\right)=\{(x-i,\lfloor\alpha x\rfloor-$ $\lfloor\alpha i\rfloor) \mid x \in \llbracket i, m+i-1 \rrbracket\}$ and $S\left(n, \beta, \gamma_{2}\right)=\{(x-j,\lfloor\beta y\rfloor-\lfloor\beta j\rfloor) \mid y \in \llbracket j, n+j-1 \rrbracket\}$. Then $w=w_{i, j}(\alpha, \beta, 0)$ verifies the conditions of the property.

Corollary 3. $\operatorname{card}\left(\mathcal{U}_{m, n}\right) \geq \operatorname{card}\left(\mathcal{S}_{m}\right) \operatorname{card}\left(\mathcal{S}_{n}\right)$. So, $\operatorname{card}\left(\mathcal{U}_{m, n}\right) \geq \frac{1}{\pi^{4}} m^{3} n^{3}+$ $O\left(m^{2} n^{2} \log (m) \log (n)\right)$.

Proof. By Property 3 , we have $\operatorname{card}\left(\mathcal{U}_{m, n}\right) \geq \operatorname{card}\left(\mathcal{S}_{m}\right) \operatorname{card}\left(\mathcal{S}_{n}\right)$ and the second assertion is a direct consequence of Property 2

Corollaries 2 and 3 imply that there exist two constant numbers $k_{1}, k_{2}$ such that $k_{1} m^{3} n^{3}<\operatorname{card}\left(\mathcal{U}_{m, n}\right)<k_{2}(m+n)^{2} m^{3} n^{3}$.

\section{Application to Local Estimators}

A digital surface is the discretization of a surface of $\mathbb{R}^{3}$. We investigate in this section the local estimators of the area of digital surface in the digital space $r \mathbb{Z}^{3}$ of resolution $r$.

The local estimator of area is obtained by associating a weight $p(w)$ to each pattern $w \in \mathcal{M}(m, n)$ of size $m \times n$ where $\mathcal{M}(m, n)$ is the set of all $m \times n$-patterns, then any digital surface $\mathcal{S}_{r}$, can be obtained by concatenation of elements in $\mathcal{M}(m, n)$ with perhaps a pattern $\varepsilon_{i}, \varepsilon_{j}^{\prime}$ of size less that $m \times n$. In other words $\mathcal{S}_{r}$ can be viewed as a bi-dimensional word on the alphabet of patterns of size less or equal to $m \times n$. If

$$
\mathcal{S}_{r}=\begin{array}{ccccc}
w_{1,1} & w_{2,1} & \cdots & w_{M, 1} & \varepsilon_{1} \\
w_{1,2} & w_{2,2} & \cdots & w_{M, 2} & \varepsilon_{2} \\
\vdots & \vdots & \cdots & \vdots & \vdots \\
w_{1, N} & w_{2, N} & \cdots & w_{M, N} & \varepsilon_{N} \\
\varepsilon_{1}^{\prime} & \varepsilon_{2}^{\prime} & \cdots & \varepsilon_{M}^{\prime} & \varepsilon_{M+1}^{\prime}
\end{array}
$$


where $w_{i, j} \in \mathcal{M}(m, n)$ for all $i, j$, then we define the area of $\mathcal{S}_{r}$ by $\mathfrak{S}_{r, m, n, p}\left(\mathcal{S}_{r}\right)=$ $r^{2} \sum_{i, j} p\left(w_{i, j}\right)$ (i.e. we neglect the contribution of the digital surfaces $\varepsilon_{i}$ and $\left.\varepsilon_{j}^{\prime}\right)$. Actually, we investigate the following problem:

Does there exist $m, n$ and $p($.$) such that for any surface \mathcal{S} \in \mathbb{R}^{3}$ the areas $\mathfrak{S}_{r, m, n, p}\left(\mathcal{S}_{r}\right)$ converge to the area of $\mathcal{S}$ where $r$ tends to 0 ? (i.e. $\mathcal{S}_{r}$ is a discretization of $\mathcal{S})$.

In this section, we study this problem for a particular class of surfaces: the set of rectangular planar regions. Moreover we suppose that the discretization operator $\delta_{r}$ restricted to these regions is in the class of the "Bresenham" discretization.

Let $a, b, c, d \in \mathbb{R}$ such that $a<b$ and $c<d$ and $0 \leq \alpha, \beta \leq 1$. Let $r>0$ be the resolution of the discrete space $r \mathbb{Z}^{3}$. Let the rectangular planar region $R=\{(x, y, \alpha x+\beta y+\gamma) \mid a \leq x \leq b$ and $c \leq y \leq d\}$. So the "Bresenham" discretization of $R$ in $r \mathbb{Z}^{3}$ is

$$
R_{r}=r\left\{\left(x, y,\left\lfloor\alpha x+\beta y+\frac{\gamma}{r}\right\rfloor\right) \mid(x, y) \in \llbracket\left\lceil\frac{a}{r}\right\rceil,\left\lfloor\frac{b}{r}\right\rfloor \rrbracket \times \llbracket\left\lceil\frac{c}{r}\right\rceil,\left\lfloor\frac{d}{r}\right\rfloor \rrbracket\right\} .
$$

We fix $m, n$ as a positive integers. As it has been explained for surfaces, the discrete region $R_{r}$ can be seen as the bi-dimensional word:

$$
R_{r}=\begin{array}{ccccc}
w_{1,1} & w_{2,1} & \cdots & w_{M_{r}, 1} & \varepsilon_{1, r} \\
w_{1,2} & w_{2,2} & \cdots & w_{M_{r}, 2} & \varepsilon_{2, r} \\
\vdots & \vdots & \cdots & \vdots & \vdots \\
w_{1, N_{r}} & w_{2, N_{r}} & \cdots & w_{M_{r}, N_{r}} & \varepsilon_{N_{r}, r} \\
\varepsilon_{1, r}^{\prime} & \varepsilon_{2, r}^{\prime} & \cdots & \varepsilon_{M_{r}, r}^{\prime} & \varepsilon_{M_{r}+1, r}^{\prime}
\end{array}
$$

where $M_{r}=\left\lfloor\frac{\left\lfloor\frac{b}{r}\right\rfloor-\left\lceil\frac{a}{r}\right\rceil+1}{m}\right\rfloor$ and $N_{r}=\left\lfloor\frac{\left\lfloor\frac{d}{r}\right\rfloor-\left\lceil\frac{c}{r}\right\rceil+1}{n}\right\rfloor$ and for all $i, j, w_{i, j}$ is a $(m, n)$ cube and $\varepsilon_{i}, \varepsilon_{j}^{\prime}$ are patterns of size less than $m \times n$.

We construct $\mathfrak{S}_{r, m, n, p}$ as the local estimator of measure by using a weight function $p: \mathcal{U}_{m, n} \rightarrow \mathbb{R}$. Then $\mathfrak{S}_{r, m, n, p}$ is defined by:

$$
\begin{aligned}
\mathfrak{S}_{r, m, n, p}\left(R_{r}\right) & =r^{2} \sum_{(i, j) \in \llbracket 1, M_{r} \rrbracket \times \llbracket 1, N_{r} \rrbracket} p\left(w_{i, j}\right) \\
& =r^{2} \sum_{w \in \mathcal{U}_{m, n}} n\left(w, R_{r}, r\right) p(w)
\end{aligned}
$$

Where $n\left(w, R_{r}, r\right)=\operatorname{card}\left(\left\{(i, j) \in \llbracket 1, M_{r} \rrbracket \times \llbracket 1, N_{r} \rrbracket \mid w_{i, j}=w\right\}\right)$ which is the number of occurrences of the pattern $w$ in the bi-dimensional word $R_{r}$ (i.e. we neglect the contributions of the $\varepsilon_{i, r}$ and $\varepsilon_{j, r}^{\prime}$ for $i \in \llbracket 1, N_{r} \rrbracket$ and $\left.j \in \llbracket 1, M_{r}+1 \rrbracket\right)$.

The central question of this section can be formulated as the following, does there exist $m, n$ and $p($.$) such that, for any rectangular planar region R$, the estimation $\mathfrak{S}_{r, m, n, p}\left(R_{r}\right)$ converges to the area of $R$ when the resolution $r$ tends to 0 ?

We will prove in this section that the response is almost everywhere no. 
Actually, we will prove that for almost all rectangular planar regions $R$, the estimation $\mathfrak{S}_{r, m, n, p}\left(R_{r}\right)$ does not converge to the area of $R$ when the resolution $r$ tends to 0 .The result of this section is an extension of the results of [17] for estimating area of rectangular planar regions of $\mathbb{R}^{3}$.

Put $D A_{m, n, p}(R)=\lim _{r \rightarrow 0} \mathfrak{S}_{r, m, n, p}\left(R_{r}\right)$.

In all the following, to simplify the notations we denote $E_{r}=\left(\llbracket\left\lceil\frac{a}{r}\right\rceil,\left\lfloor\frac{b}{r}\right\rfloor \rrbracket \times\right.$ $\left.\llbracket\left\lceil\frac{c}{r}\right\rceil,\left\lfloor\frac{d}{r}\right\rfloor \rrbracket\right) \cap\left(\left(m \mathbb{Z}+\left\lceil\frac{a}{r}\right\rceil\right) \times\left(n \mathbb{Z}+\left\lceil\frac{c}{r}\right\rceil\right)\right)$ and $S_{r}=\left(\left\lfloor\frac{b}{r}\right\rfloor-\left\lceil\frac{a}{r}\right\rceil+1\right)\left(\left\lfloor\frac{d}{r}\right\rfloor-\left\lceil\frac{c}{r}\right\rceil+1\right)$

Definition 8. The non-overlapping frequency $F_{r}^{\alpha, \beta, \gamma, a, b, c, d}$ of a pattern $w$ of size $m \times n$ in $R_{r}$ is defined by:

$$
F_{r}^{\alpha, \beta, \gamma, a, b, c, d}=\frac{\operatorname{card}\left(\left\{(x, y) \in E_{r} \mid w_{x, y}\left(\alpha, \beta, \frac{\gamma}{r}\right)=w\right\}\right)}{S_{r}}
$$

Lemma 1. Let $\alpha, \beta \in[0,1]$ such that $\alpha$ or $\beta$ is irrational, $\gamma, a, b, c, d \in \mathbb{R}$, $w \in \mathcal{C}_{m, n, \alpha, \beta}$. Then

$$
F^{\alpha, \beta, \gamma, a, b, c, d}=\lim _{r \rightarrow 0} F_{r}^{\alpha, \beta, \gamma, a, b, c, d}=\frac{1}{m n} \operatorname{freq}_{\alpha, \beta}(w)
$$

In particular $F^{\alpha, \beta, \gamma, a, b, c, d}$ does not depend on $\gamma, a, b, c$, and $d$.

Proof.

$$
\begin{aligned}
F^{\alpha, \beta, \gamma, a, b, c, d} & =\lim _{r \rightarrow 0} \frac{\operatorname{card}\left(\left\{(x, y) \in E_{r} \mid w_{x, y}\left(\alpha, \beta, \frac{\gamma}{r}\right)=w\right\}\right)}{S_{r}} \\
& =\lim _{r \rightarrow 0} \frac{\operatorname{card}\left(\left\{(x, y) \in E_{r} \mid\left\langle\alpha x+\beta y+\frac{1}{r} \gamma\right\rangle \in I^{\alpha, \beta}(w)\right\}\right)}{S_{r}}
\end{aligned}
$$

So, if we take $p=m, q=n, \gamma_{r}=\frac{1}{r} \gamma$ and $I=I^{\alpha, \beta}(w)$ in Theorem 4 of the Appendix A [1], then we have $F^{\alpha, \beta, \gamma, a, b, c, d}=\frac{1}{m n} \mu\left(I^{\alpha, \beta}(w)\right)=\frac{1}{m n} \operatorname{freq}_{\alpha, \beta}(w)$ because by Proposition 2 overfreq $_{\alpha, \beta, \gamma}(w)=\mu\left(I^{\alpha, \beta}(w)\right)$

Theorem 3. Let $\mathcal{O}$ be a connected component of $[0,1]^{2} \backslash E_{m, n}$. Then there exist $u, v, t \in \mathbb{R}$ such that $D A_{m, n, p}(R)=(b-a)(d-c)(u \alpha+v \beta+t)$ for all rectangular planar regions $R=\{(x, y, \alpha x+\beta y+\gamma) \mid a \leq x \leq b$ and $c \leq y \leq d\}$ such that $\alpha, \beta \in \mathcal{O}$ and $\alpha$ or $\beta$ is irrational.

In other words, $D A_{m, n, p}($.$) is an affine function in (\alpha, \beta)$ for $(\alpha, \beta) \in\left(\mathcal{O} \backslash \mathbb{Q}^{2}\right)$.

Proof. By (1) and (2) we have:

$$
\begin{aligned}
\mathfrak{S}_{r, m, n, p}\left(R_{r}\right) & =r^{2} \sum_{1 \leq i \leq M_{r}} \sum_{1 \leq j \leq N_{r}} p\left(w_{i, j}\right) \\
& =r^{2} \sum_{w \in \mathcal{U}_{m, n}} n\left(w, R_{r}, r\right) p(w)
\end{aligned}
$$

where $n\left(w, R_{r}, r\right)=\operatorname{card}\left(\left\{(x, y) \in\left(\left(\left(m \mathbb{Z}+\left\lceil\frac{a}{r}\right\rceil\right) \times\left(n \mathbb{Z}+\left\lceil\frac{c}{r}\right\rceil\right)\right) \cap\left(\left[\left\lceil\frac{a}{r}\right\rceil,\left[\left\lfloor\frac{b}{r}\right\rfloor\right] \times\right.\right.\right.\right.\right.$ $\left.\left.\left[\left\lceil\frac{c}{r}\right\rceil,\left[\left\lfloor\frac{d}{r}\right\rfloor\right]\right) \mid w_{x, y}\left(\alpha, \beta, \frac{\gamma}{r}\right)=w\right\}\right)$ which is the number of occurrences of the pattern $w$ in the bi-dimensional word $R_{r}$. So, 


$$
\begin{aligned}
D A_{m, n, p}(R) & =\lim _{r \rightarrow 0} r^{2} \sum_{w \in \mathcal{U}_{m, n}} n\left(w, R_{r}, r\right) p(w) \\
& =\lim _{r \rightarrow 0} r^{2} S_{r} \sum_{w \in \mathcal{U}_{m, n}} \frac{n\left(w, R_{r}, r\right)}{S_{r}} p(w) \\
& =(b-a)(d-c) \sum_{w \in \mathcal{U}_{m, n}} \frac{1}{m n} \operatorname{freq}_{\alpha, \beta}(w) p(w) \text { (By Lemma 1) }
\end{aligned}
$$

So, according to Theorem 2, $D A_{m, n, p}($.$) is an affine function in (\alpha, \beta)$ for $(\alpha, \beta) \in$ $\left(\mathcal{O} \backslash \mathbb{Q}^{2}\right)$

Corollary 4. The set of $(\alpha, \beta) \in\left([0,1]^{2} \backslash E_{m, n}\right)$ such that $\alpha$ or $\beta$ is irrational and $D A_{m, n, p}(R)=\operatorname{area}(R)$ is a negligible (relatively to the Lebesgue measure on the Euclidean space) where for $a, b, c, d \in \mathbb{R}, R=\{(x, y, \alpha x+\beta y+\gamma) \mid a \leq$ $x \leq b$ and $c \leq y \leq d\}$.

Proof. We consider a connected component $\mathcal{O}$ of $[0,1]^{2} \backslash E_{m, n}$. By Theorem 3 , there exist $u, v, t \in \mathbb{R}$ such that the estimated area of the rectangular planar region $R$ is $D A_{m, n, p}(R)=(b-a)(d-c)(u \alpha+v \beta+t)$ for $\alpha$ or $\beta$ is irrational. The exact area of $R$ is area $(R)=(b-a)(d-c) \sqrt{1+\alpha^{2}+\beta^{2}}$. So we have:

$D A_{m, n, p}(R)=\operatorname{area}(R) \Longleftrightarrow(u \alpha+v \beta+t)^{2}=1+\alpha^{2}+\beta^{2}$

Which is equivalent to $\left(u^{2}-1\right) \alpha^{2}+\left(v^{2}-1\right) \beta^{2}+2(u v \alpha \beta+u t \alpha+v t \beta)+t^{2}-1=0$ But, the last equation corresponds to an object of Lebesgue measure greater than 0 only when $u^{2}-1=0, v^{2}-1=0, t^{2}-1=0, u v=0, u t=0$ and $v t=0$ which never happens. So, the last equation corresponds to a curve in $\mathbb{R}^{2}$ (which is the intersection of conic and the region $\mathcal{O})$ and thus, for $(\alpha, \beta) \in \mathcal{O}$, the estimated area can be equal to the exact area for only $(\alpha, \beta)$ in a set included in the intersection of a conic and the region $\mathcal{O}$ which corresponds to a negligible set.

But, $[0,1]^{2} \backslash E_{m, n}$ contains only a finite number of connected components. Thus, the set of $(\alpha, \beta) \in\left([0,1]^{2} \backslash\left(E_{m, n} \cup \mathbb{Q}^{2}\right)\right)$ such that the estimated area is equal to the exact area is a negligible set because it is a finite union of negligible sets.

Corollary 5. For any $m, n \in \mathbb{N}^{*}$ and any weight function $p($.$) the set of (\alpha, \beta) \in$ $[0,1]^{2}$ such that the rectangular planar region $R=\{(x, y, \alpha x+\beta y+\gamma) \mid a \leq x \leq$ $b$ and $c \leq y \leq d\}$ (where $\gamma, a, b, c, d \in \mathbb{R}$ ) satisfies area $(R)=D A_{m, n, p}(R)$ is a negligible set. So, for any $m, n \in \mathbb{N}^{*}$ and any weight function $p($.$) , for$ all rectangular planar regions $R$ with the parameters $\alpha, \beta \in[0,1]$, we have $\operatorname{area}(R) \neq D A_{m, n, p}(R)$ almost everywhere.

Proof. By Corollary 5, we have, for almost all rectangular planar regions $R$ with parameters $(\alpha, \beta) \in\left([0,1]^{2} \backslash E_{m, n}\right)$ area $(R) \neq D A_{m, n, p}(R)$. But $\mathbb{Q}^{2}$ is infinite countable set and $E_{m, n}$ is a finite set of straight lines. So $E_{m, n} \cup \mathbb{Q}^{2}$ is a negligible set. So, for all rectangular planar regions $R$ with the parameters $\alpha, \beta \in[0,1]$, $\operatorname{area}(R) \neq D A_{m, n, p}(R)$ almost everywhere 


\section{Conclusion}

In this paper we have seen that the frequencies of the $(m, n)$-cubes of digital planes are given by a continuous piecewise affine function in the slopes of the digital planes. This has consequences on the combinatorics of $(m, n)$-cubes, in particular on the asymptotic behavior of the number of $(m, n)$-cubes when $m$ and $n$ tend to infinity.

Moreover it has also consequences on local estimators of area as it permits to prove that any local estimator of area is never multigrid-convergent: for almost all region of plane it does not converge to the true area. This result is a generalization of a result in dimension two proved in [17. Actually we can prove with the same technics that this result is true for the equivalent notions for any finite dimension.

\section{Acknowledgment}

This work was supported by the IRMC program.

\section{References}

1. Daurat, A., Tajine, M., Zouaoui, M.: About the frequencies of some patterns in digital planes. Application to area estimators - extended version (preprint, 2007), http://hal .archives-ouvertes.fr/hal-00174960

2. Forchhammer, S.: Digital plane and grid point segments. Comput. Vis. Graph. Image Process 47, 373-384 (1989)

3. Françon, J., Schramm, J.M., Tajine, M.: Recognizing arithmetic straight lines and planes. In: Miguet, S., Ubéda, S., Montanvert, A. (eds.) DGCI 1996. LNCS, vol. 1176, pp. 141-150. Springer, Heidelberg (1996)

4. Schramm, J.M.: Coplanar tricubes. In: Ahronovitz, E. (ed.) DGCI 1997. LNCS, vol. 1347, pp. 87-98. Springer, Heidelberg (1997)

5. Reveillès, J.P.: Combinatorial pieces in digital lines and planes. In: Proc. SPIE Vision Geometry IV, vol. 2573, pp. 23-34 (1995)

6. Gérard, Y.: Contribution à la Géométrie Discrète. $\mathrm{PhD}$ thesis, Université d'Auvergne, Clermont-Ferrand (1999)

7. Gérard, Y.: Local Configurations of Digital Hyperplanes. In: Bertrand, G., Couprie, M., Perroton, L. (eds.) DGCI 1999. LNCS, vol. 1568, pp. 65-95. Springer, Heidelberg (1999)

8. Vittone, J., Chassery, J.M. (n,m)-Cubes and Farey Nets for Naive Planes Understanding. In: Bertrand, G., Couprie, M., Perroton, L. (eds.) DGCI 1999. LNCS, vol. 1568, pp. 76-90. Springer, Heidelberg (1999)

9. Vittone, J.: Caractérisation et reconnaissance de droites et de plans en géométrie discrète. PhD thesis, Université Joseph Fourier, Grenoble (1999)

10. Vuillon, L.: Combinatoire des motifs d'une suite sturmienne bidimensionnelle. Theoret. Comput. Sci. 209, 261-285 (1998)

11. Vuillon, L.: Local configurations in a discrete plane. Bull. Belg. Math. Soc. Simon Stevin 6(4), 625-636 (1999) 
12. Brimkov, V.E., Andres, E., Barneva, R.P.: Object discretizations in higher dimensions. Pattern Recogn. Lett. 23(6), 623-636 (2002)

13. Veelaert, P.: Digital planarity of rectangular surface segments. IEEE Trans. Pattern Anal. Mach. Intell. 16(6), 647-652 (1994)

14. Brimkov, V., Coeurjolly, D., Klette, R.: Digital planarity - a review. Discrete Appl. Math. 155(4), 468-495 (2007)

15. Lindblad, J.: Surface area estimation of digitized 3d objects using weighted local configurations. Image Vis. Comput. 23(2), 111-122 (2005)

16. Kenmochi, Y., Klette, R.: Surface area estimation for digitized regular solids. In: Proc. SPIE, Vision Geometry IX, vol. 4117, pp. 100-111 (2000)

17. Tajine, M., Daurat, A.: On Local Definitions of Length of Digital Curves. In: Nyström, I., Sanniti di Baja, G., Svensson, S. (eds.) DGCI 2003. LNCS, vol. 2886, pp. 114-123. Springer, Heidelberg (2003)

18. Coeurjolly, D., Sivignon, I., Dupont, F., Feschet, F., Chassery, J.M.: On digital plane preimage structure. Discrete Appl. Math. 151(1-3), 78-92 (2005)

19. Berthé, V., Fiorio, C., Jamet, D., Philippe, F.: On some applications of generalized functionality for arithmetic discrete planes. Image Vis. Comput. 25(10), 1671-1684 (2007)

20. Berenstein, C., Lavine, D.: On the number of digital straight line segments. IEEE Trans. On Pattern Analysis and Machine Intelligence 10(6), 880-887 (1988)

21. Mignosi, F.: On the number of factors of Sturmian words. Theoret. Comput. Sci. 82(1), 71-84 (1991) 